\title{
Different invasion phenotypes of Campylobacter isolates in Caco-2 cell monolayers
}

\author{
PHILIPPA HARVEY, THIERRY BATTLE and STEVE LEACH \\ Centre for Applied Microbiology and Research, Porton Down, Salisbury, Wiltshire SP4 OJG
}

\begin{abstract}
The pathogenesis of campylobacter enteritis is not well understood, but invasion into and translocation across intestinal epithelial cells may be involved in the disease process, as demonstrated for a number of other enteric pathogens. However, the mechanisms involved in these processes are not clearly defined for campylobacters. In this study, isolates were compared quantitatively in established assays with the enterocyte-like cell line, Caco-2, to determine the extent to which intracellular invasion contributes to translocation across epithelial cell monolayers, and whether isolates vary in this respect. Ten fresh Campylobacter isolates were compared and shown to differ in invasiveness by a factor of 10-fold by following their recovery from gentamicin-treated Caco-2 cells grown on nonpermeable tissue-culture wells. Four of these isolates with contrasting invasive ability were also shown to vary in their ability to translocate across Caco-2 cells grown on semipermeable Transwell inserts by a factor $>10$. However, translocation did not quantitatively correlate with the intracellular invasiveness of these isolates. Isolate no. 9752 was poorly invasive but had modest translocation ability, isolate no. 10392 was very invasive but did not translocate significantly and remained within the monolayer, isolate no. 9519 both translocated and invaded well, whereas, isolate no. 235 translocated very efficiently but was poorly invasive. Isolate no. 9519 also uniquely caused a transitory flattening of the Caco-2 cells and a possible drop in trans-epithelial electrical resistance (TEER) of the Transwell monolayers, whereas isolate no. 235 did not show these effects. Together these data demonstrate that there are significantly different 'invasion' phenotypes among Campylobacter strains involving different degrees of intracellular invasion, and either different rates of transcellular trafficking or, alternatively, paracellular trafficking.
\end{abstract}

\section{Introduction}

Enteric bacteria vary greatly in their interactions with mammalian intestinal cells in the process of causing disease in the host [1-3]. Many of these pathogens have to evade the host defence system and penetrate the epithelial cell barrier to cause disease. This allows these pathogens to exert their physiopathological effects and to interact with the mucosa-associated lymphoid tissues [4]. The epithelial barrier can be infiltrated by these invasive enteropathogens by various methods of invasion or translocation, or both. Translocation involves the pathogen passing through the enterocyte cell layer to gain access to the underlying tissues. The ability to translocate in this way has been demonstrated for many enteroinvasive pathogens,

Date received 21 May 1998; revised version accepted 27 Aug. 1998.

Corresponding author: Miss P. Harvey (e-mail: philippa. harvey@camr.org.uk). including Salmonella spp. [5], enteropathogenic Escherichia coli [6] and Listeria monocytogenes [7].

Enteric campylobacters, particularly Campylobacter jejuni and $C$. coli, are a major cause of enteric disease, resulting in either severe inflammatory or mild non-inflammatory diarrhoea. The extent of the disease is thought to be influenced by a number of factors, including the virulence determinants of the pathogen such as outer-membrane proteins, toxins, flagella and pili, the expression of which may differ between strains [8]. C. jejuni is also thought to adhere to, colonise and invade the small intestinal and colonic mucosae [9]. Penetration of the intestinal mucosa by intracellular invasion has been reported in infected monkeys. This resulted in inflammation, infiltration of the lamina propria by neutrophils and bacteraemia, which suggests that invasion is an important pathogenic mechanism for $C$. jejuni [10]. Additional invasive pathogenic mechanisms may also be involved in campylobacter infections, as observations by 
electron microscopy have demonstrated $C$. jejuni translocating both through and between epithelial cells in culture, with $C$. jejuni located in junctional spaces in some instances $[11,12]$. This suggests that both transcellular and paracellular routes are important in campylobacter invasion, but the relative importance of these different routes is not clear.

Various cell culture systems, including HEp-2 and INT407, have been used widely to study the disease mechanisms of campylobacters [12-14]. However, the enterocyte-like Caco- 2 cell line, derived from a human colonic carcinoma, is probably more relevant because it represents more closely the site of invasion in vivo, in both architecture and function [15]. The polarised Caco-2 cells differentiate to give distinct apical and basolateral surfaces, possessing microvilli and markers characteristic of small intestinal cells [16]. Caco-2 cells grown on non-permeable tissue culture wells have been used previously for bacterial invasion studies [17] including those of Campylobacter spp. [18]. However, when grown on porous membranes, the Caco-2 cells probably model the columnar intestinal barrier more closely, with the cells and intercellular tight junctions separating the medium surrounding the apical surface from that surrounding the basolateral surface [16]. This model has been used to investigate epithelial cell translocation for a number of pathogens including Salmonella spp. [5] and C. jejuni $[11,18]$.

Although previous studies have demonstrated the ability of $C$. jejuni to translocate across Caco-2 cell monolayers $[8,11,19]$, neither the mechanism by which campylobacters invades these cells, nor the relative contribution of paracellular translocation to epithelial penetration in these systems is completely understood. Previous studies [18] have demonstrated that more invasive and translocating strains may correspond to those that cause colitis and more severe disease. However, the relative importance of the different putative translocation mechanisms has not been clearly quantified. Four different possible interactions between campylobacters and epithelial cells have been proposed [8], including non-invasion, invasion, invasion with translocation and translocation without invasion.

In order to assess the relative importance of the different potential translocation mechanisms used by Campylobacter spp., both the translocation and the invasive abilities of isolates of the pathogen in Caco-2 cells have been compared quantitatively. In the translocation studies, changes in the trans-epithelial electrical resistance and the gross morphology of the monolayers following infection were also monitored. The data provide support for at least three quantitatively distinct modes of translocation across epithelial cell monolayers, which are dependent to very different extents on intracellular invasion and the modification of monolayer integrity and structure.

\section{Materials and methods}

\section{Bacteria}

Fresh isolates of Campylobacter spp. were provided by Professor T. Humphrey, Exeter PHL (C. jejuni: 206, 235, 9519, 9752, 10392, CH1, CH5; C. coli: 247, 799) and Dr R. Owen, CPHL, 61 Colindale Avenue, London (C. jejuni: C677). All isolates were from clinical cases of human campylobacteriosis except for $\mathrm{CH} 1$ and $\mathrm{CH} 5$ which were poultry isolates. All isolates had been minimally passaged and were subsequently maintained on bead stocks (Prolab) at $-70^{\circ} \mathrm{C}$. They were revived for each experiment by plating on to Columbia Blood Agar (bi-layer plates with defibrinated horse blood $10 \%$ $\mathrm{v} / \mathrm{v}, \mathrm{CBA}$, Oxoid), which was incubated at $37^{\circ} \mathrm{C}$ in a $\mathrm{CO}_{2}$ incubator (ASSAB, Don Whitley; $\mathrm{O}_{2} 4 \%$ and $\mathrm{CO}_{2}$ $5 \%$ ). Growth was harvested from single plates to inoculate $10-\mathrm{ml}$ volumes of Cpmod4 broth [20] in $100-\mathrm{ml}$ conical flasks.

Cultures were incubated at $37^{\circ} \mathrm{C}$ with shaking (LH orbital shaker, $150 \mathrm{rpm}$ ) in a continuous supply of gas containing $\mathrm{O}_{2} 5 \% \mathrm{v} / \mathrm{v}$ in $\mathrm{N}_{2}$. After $10 \mathrm{~h}$, the cultures had entered late $\log$ phase and $1 \mathrm{ml}$ of each was used to inoculate $10 \mathrm{ml}$ of fresh Cpmod4 broth. The flasks were returned to the incubator and growth continued for a further $12 \mathrm{~h}$ under the same conditions. Cells were harvested by centrifugation (MSE microfuge, $11000 \mathrm{rpm}, 5 \mathrm{~min}$ ) and resuspended to an appropriate cell density in tissue culture medium (see below).

\section{Cultured epithelial cells}

Caco-2 cells were maintained in minimal essential medium (MEM) with non-essential amino acids (NEAA), glutamine and fetal calf serum (FCS) $10 \%$ $\mathrm{v} / \mathrm{v}$, without antibiotics. Cells were grown routinely in flasks at $37^{\circ} \mathrm{C}$ in humidified air with $\mathrm{CO}_{2} 5 \%$. The Caco- 2 cells were seeded at $2 \times 10^{5}$ cells $/ \mathrm{ml}$ into 24 well tissue culture plates (Costar, MA, USA). These were maintained for 7 days with three media changes to reach confluency, before use.

\section{Invasion assay}

The assay was based on that of Everest et al. [18]. Each well of Caco-2 cells was infected with $0.5 \mathrm{ml}$ of a suspension of $10^{8}$ campylobacter cells $/ \mathrm{ml}$ in $\mathrm{MEM}+\mathrm{NEAA}+\mathrm{FCS} 1 \%$, as estimated by direct counts. The wells were incubated at $37^{\circ} \mathrm{C}$ in a $\mathrm{CO}_{2}$ incubator. After 3 or $6 \mathrm{~h}$ the wells were treated to determine the number of intracellular bacteria. The bacterial suspension was removed and the monolayer was incubated for $2 \mathrm{~h}$ with MEM containing gentamicin $250 \mu \mathrm{g} / \mathrm{ml}$ to kill the extracellular bacteria. The monolayers were washed three times with PBS to remove the gentamicin. The intracellular bacteria were released by lysing the monolayers with ice-cold Triton $\mathrm{X}-1001 \% \mathrm{v} / \mathrm{v}$ in PBS. Viable counts were then determined by serial dilutions in phosphate-buffered 
saline (PBS) and plating on to CBA. The viable count of the inoculum applied to the monolayers was determined and the number of intracellular bacteria was expressed as a proportion of this inoculum. Invasion assays were performed in duplicate on three separate occasions with each isolate.

\section{Preparation of Transwell units}

The assay was based on that of Finlay and Falkow [5]. Caco- 2 cells were utilised between passages 8 and 14 . The cells were maintained in MEM with FCS $10 \% \mathrm{v} / \mathrm{v}$, NEAA and glutamine, without antibiotics. The Caco-2 cells were trypsinised and $0.5 \mathrm{ml}$ of a $1 \times 10^{5}$ cells $/ \mathrm{ml}$ suspension in tissue-culture media, as determined by direct counts, was added to each Transwell insert ( $0.4 \mu \mathrm{m}$ pore, $12 \mathrm{~mm}$ diameter, collagen-coated polycarbonate; Costar). Each insert was placed in a well containing $1.2 \mathrm{ml}$ of fresh MEM + NEAA+ FCS $1 \%+$ glutamine. The 12 -well tissue culture plates were then incubated at $37^{\circ} \mathrm{C}$ as described above, with apical and basolateral medium changes every 3 days. The monolayers were used after incubation for 7 days, having reached confluence at 3 days.

\section{Translocation assay}

C. jejuni isolates 235, 9519, 9752 and 10392 (chosen for their contrasting invasive abilities) were grown in batch culture as described above and a population of $10^{8}$ cells $/ \mathrm{ml}$ of each was prepared in MEM + NEAA+ FCS $1 \% \mathrm{v} / \mathrm{v}$, as estimated by direct counts. The basolateral medium was removed and replaced with $1.2 \mathrm{ml}$ of tissue culture medium containing FCS $1 \%$ $\mathrm{v} / \mathrm{v}$ in each well. An inoculum of $0.5 \mathrm{ml}$ of the bacterial suspension was added to the Caco- 2 cells in duplicate Transwell inserts. The plates were incubated at $37^{\circ} \mathrm{C}$ as described previously and Transwell inserts were removed for sampling at regular intervals. Translocation assays were performed with each isolate on three separate occasions.

\section{Quantification of bacterial translocation}

Samples $(100 \mu 1)$ of tissue culture medium were removed from the apical and basolateral compartments. The numbers of cfu were determined by serial dilutions in PBS and plating on CBA plates. The number of $C$. jejuni passing through the monolayer at each time point was expressed as a proportion of the bacterial count in the upper chamber.

\section{Measurements of trans-epithelial electrical resistance (TEER) across infected Caco-2 monolayers}

The Transwell inserts were removed at specific time intervals and the TEER was measured with a MilicellERS apparatus (Millipore, Bedford, MA, USA) with the 'STX-type' electrode. Different Transwells were set up in duplicate and used for each time point in order to avoid damaging the monolayers by repeatedly sampling single wells. The TEER of the Transwell inserts was determined after replacement of the culture medium with PBS on both sides of the membrane. The probe legs were placed either side of the insert and each TEER value was measured in triplicate and corrected for filter area.

Before the start of each experiment, control values of the TEER were determined on representative uninfected Transwell inserts containing confluent Caco-2 cell monolayers. Control values averaged 430 SEM 10 $\Omega \mathrm{cm}^{2}(95 \% \mathrm{CI} ; \mathrm{n}=36)$. The TEER of the experimental wells was measured only after the required period of incubation with $C$. jejuni, as repeated measurements on individual wells themselves was likely to interfere with monolayer integrity and the TEER. The effect of the C. jejuni isolates on the resistance across the monolayer was determined by subtracting the control value from the experimental values recorded at each time point, giving a base-line corrected TEER for uninfected monolayers of $0 \Omega \mathrm{cm}^{2}$.

\section{Determination of intracellular invasion of the Transwell insert monolayers by $C$. jejuni}

The number of intracellular $C$. jejuni within the monolayer was determined by repeating the translocation assay with the following modifications. C. jejuni isolates were applied to the monolayers as before and designated wells were sampled after 2,4 and $6 \mathrm{~h}$. The basolateral medium was replaced with fresh MEM + NEAA + FCS $1 \%$ and that in the Transwell insert with $500 \mu \mathrm{l}$ of MEM + NEAA + FCS $1 \%$ containing gentamicin $250 \mu \mathrm{g} / \mathrm{ml}$. The wells were returned to the incubator for a further $2 \mathrm{~h}$ to kill extracellular bacteria $[14,17,18]$.

After the incubation period the monolayers were washed twice with PBS, before lysis with ice-cold Triton X-100 $1 \%$ in PBS. Viable counts were determined by serial dilutions in PBS and plating on to CBA. The viable count of each inoculum applied to the monolayers was also determined. Counts of intracellular bacteria were expressed as a proportion of the inoculum in each case, in order to make direct comparison with the original invasion assay data.

\section{Examination of infected monolayers by light microscopy}

Transwell inserts from some of the experimental Transwells infected with isolates nos. 235 and 9519 were removed at 2 and $6 \mathrm{~h}$ and fixed overnight in glutaraldehyde $2 \%$ in $0.1 \mathrm{M}$ sodium phosphate buffer. Uninfected control wells were also fixed by the same process. Membranes were embedded in paraffin wax, sectioned and stained with haemotoxylin and eosin for light microscopy. 


\section{Statistical analysis}

The data sets were expressed as means and SEMs were calculated. A non-parametric test, Kruskal-Wallis, was used to determine the significance of the differences in the invasion or translocation abilities between the Campylobacter isolates. Paired comparisons of isolates were made by the Mann-Whitney U test. A $p$ value of $<0.05$ was considered significant.

\section{Results}

Invasiveness of Campylobacter isolates into Caco2 cell monolayers on non-permeable supports

During the $6 \mathrm{~h}$ invasion assay, the proportion of the bacterial inoculum within the Caco-2 cells and protected from the gentamicin increased with time for all the isolates examined (Fig. 1). The two $C$. coli isolates did not differ in their invasive potential when compared with the $C$. jejuni isolates. However, after $6 \mathrm{~h}$, the isolates differed significantly from one another in their invasiveness, as determined by Kruskal-Wallis test $(p=0.002)$. Two groups of isolates could be discerned; one group (CH1, 799, 9519, 10392, C677, 206) was considerably more invasive than the other (CH5, 235, 247, 9752). Furthermore, the kinetics of invasion also differed between isolates, some showing a much greater proportional increase in invasion between 3 and $6 \mathrm{~h}$ than between 0 and $3 \mathrm{~h}$ (e.g., nos. 9519 and 10392). In contrast, isolate no. 206 invaded the monolayers to a higher level by $3 \mathrm{~h}$.

\section{Bacterial translocation across Caco-2 cell monolayers}

The four isolates showed some slight variation in their growth in the MEM + FCS $1 \%$, the growth rate constants averaging $1.3 / \mathrm{h}$ for isolate no. $9519,1.1 / \mathrm{h}$ for no. $235,0.89 / \mathrm{h}$ for no. 9752 and $1.2 / \mathrm{h}$ for no. 10392. Each of the four isolates translocated across the monolayers into the lower chamber throughout the $6 \mathrm{~h}$ of the assay, but to significantly different extents. Comparison of the isolates showed that a higher proportion of $C$. jejuni 235 was recovered in the lower chamber at $6 \mathrm{~h}$ (Fig. 2) than any of the other isolates. Lower proportions of isolates nos. 9519 and 9752 were recovered over the same time course, with $75 \%$ and $85 \%$ fewer cells, respectively, translocating as compared with isolate no. 235 at $6 \mathrm{~h}$. In contrast, isolate no.10392 showed a much poorer ability to translocate, with $99.7 \%$ fewer cells translocating than isolate no. 235 by $6 \mathrm{~h}$. Analysis of the data by the Kruskal-Wallis test demonstrated that the isolates differed significantly in their abilities to translocate $(p=0.002)$. Individual pairs which differed significantly were no. 10392 versus no. $9752(\mathrm{p}=0.02)$, no. 10392 versus no. 235 $(p=0.05)$, no. 9752 versus no. $9519(p=0.005)$, no. 9519 versus no. $235(\mathrm{p}=0.005)$ as determined by the Mann-Whitney U test.

\section{Changes in TEER of infected monolayers}

Infection of the polarised Caco-2 monolayers with the Campylobacter isolates caused some responses in the TEER across the monolayer (Fig. 3). However, the variation within the individual data sets prevented statistically significant conclusions being drawn from the measurements. Nevertheless, one interesting trend was observed. Infection with isolate no. 9519 resulted in a rapid decline in TEER across the monolayer by $2 \mathrm{~h}$, followed by recovery over the remaining $4 \mathrm{~h}$, this being the only consistent trend between replicate experiments. With the other isolates, the TEER appeared to change less dramatically, showing only

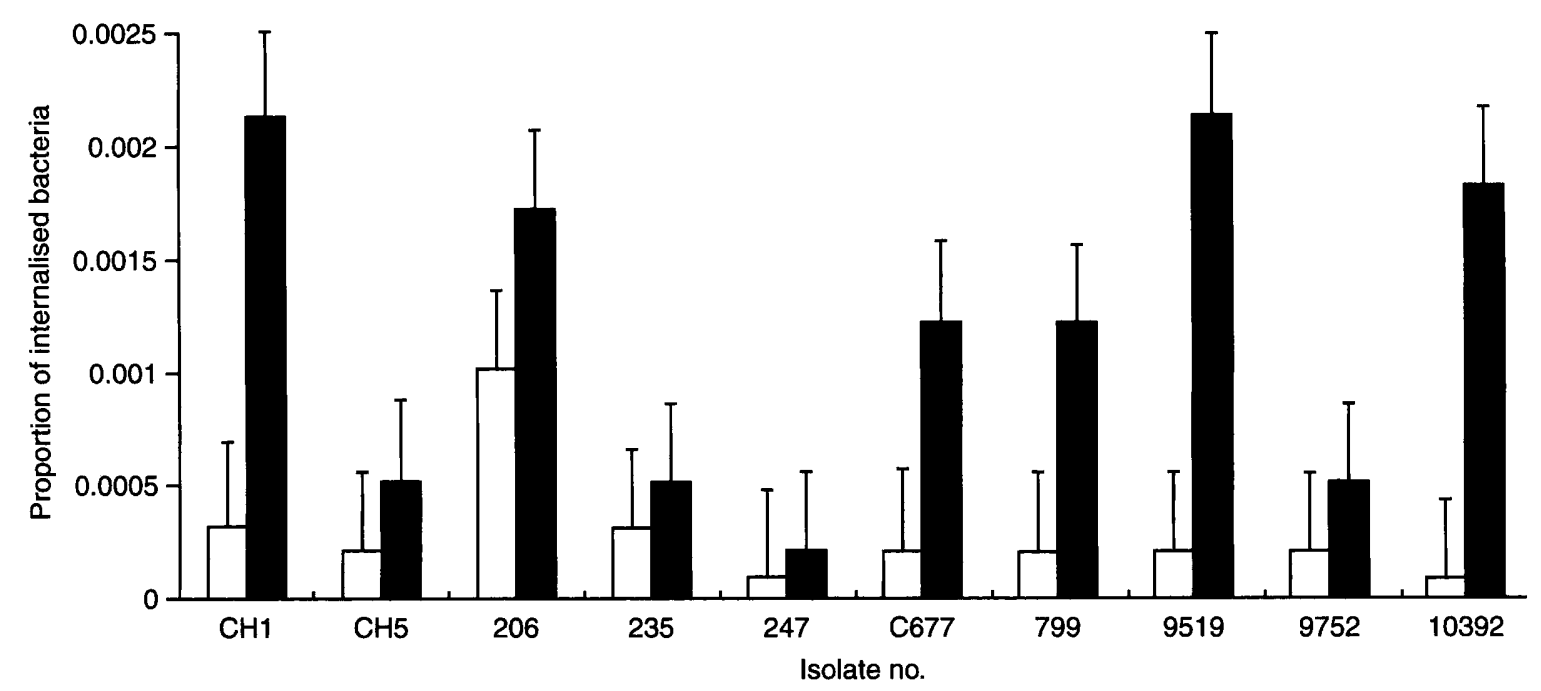

Fig. 1. The invasiveness of different Campylobacter isolates in Caco-2 cell monolayers cultured on non-permeable supports at $3 \mathrm{~h}(\square)$ and $6 \mathrm{~h}(\mathbf{\square})$. Data represent the proportion of the inoculum internalised by the Caco-2 cells. Bars represent means and SEM (12 separate determinations from three separate experiments). Differences between isolates were highly significant $(\mathrm{p}=0.002$, Kruskal-Wallis non-parametric analysis). 


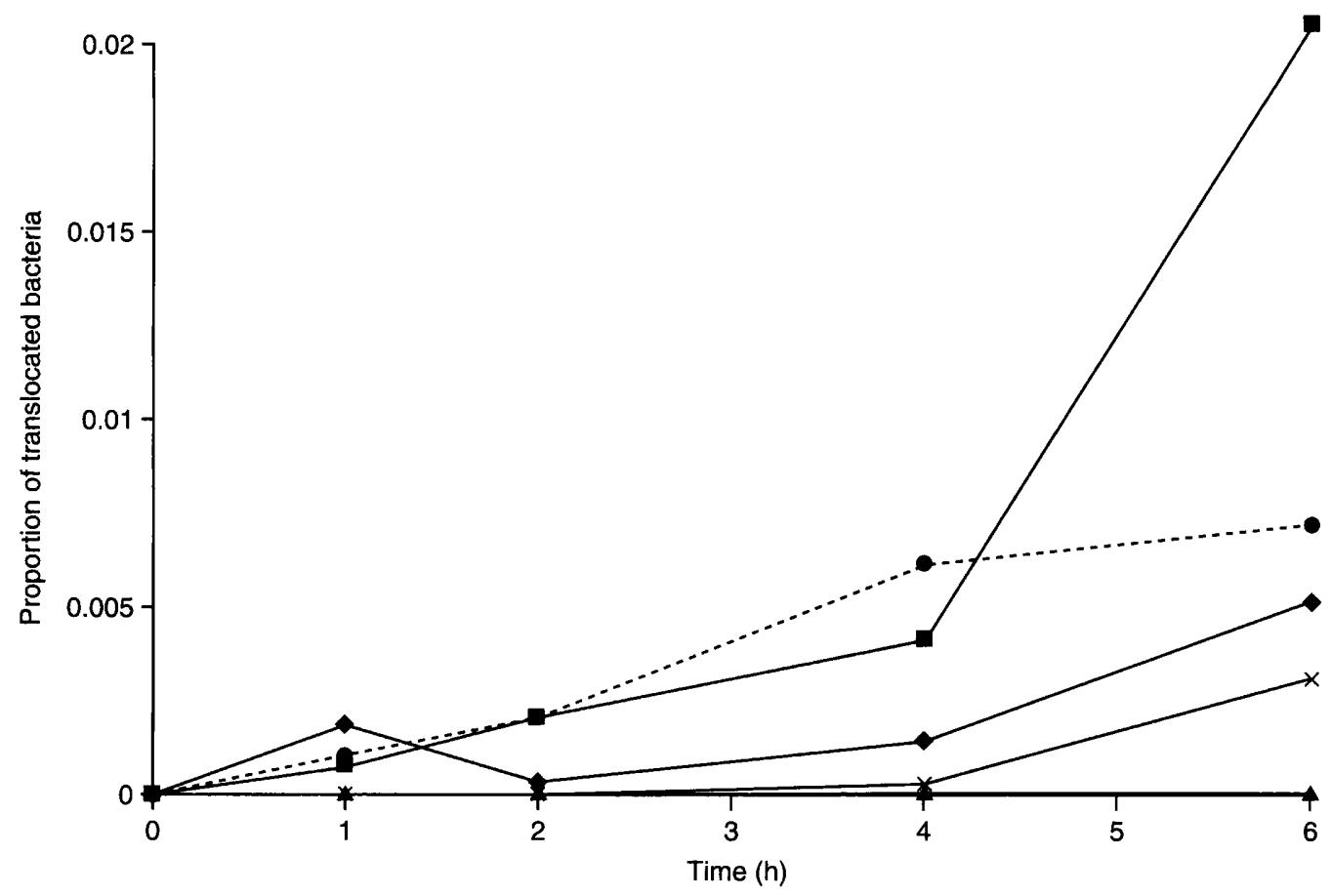

Fig. 2. Comparison of the ability of four isolates of $C$. jejuni of differing invasiveness to translocate across polarised Caco-2 monolayers cultured on semi-permeable supports. Isolates: -7 , no. $235 ;-\bullet-$, no. 9519 ; $\rightarrow-$, no. 9752 ; $A$, no. 10392; - - 10392 (data $\times 100)$. Data represent the proportion of the inoculum recovered in the lower chamber. Data points are mean values from six separate determinations from three separate experiments. Differences between isolates were highly significant $(\mathrm{p}=0.0002$, Kruskal-Wallis non-parametric analysis).

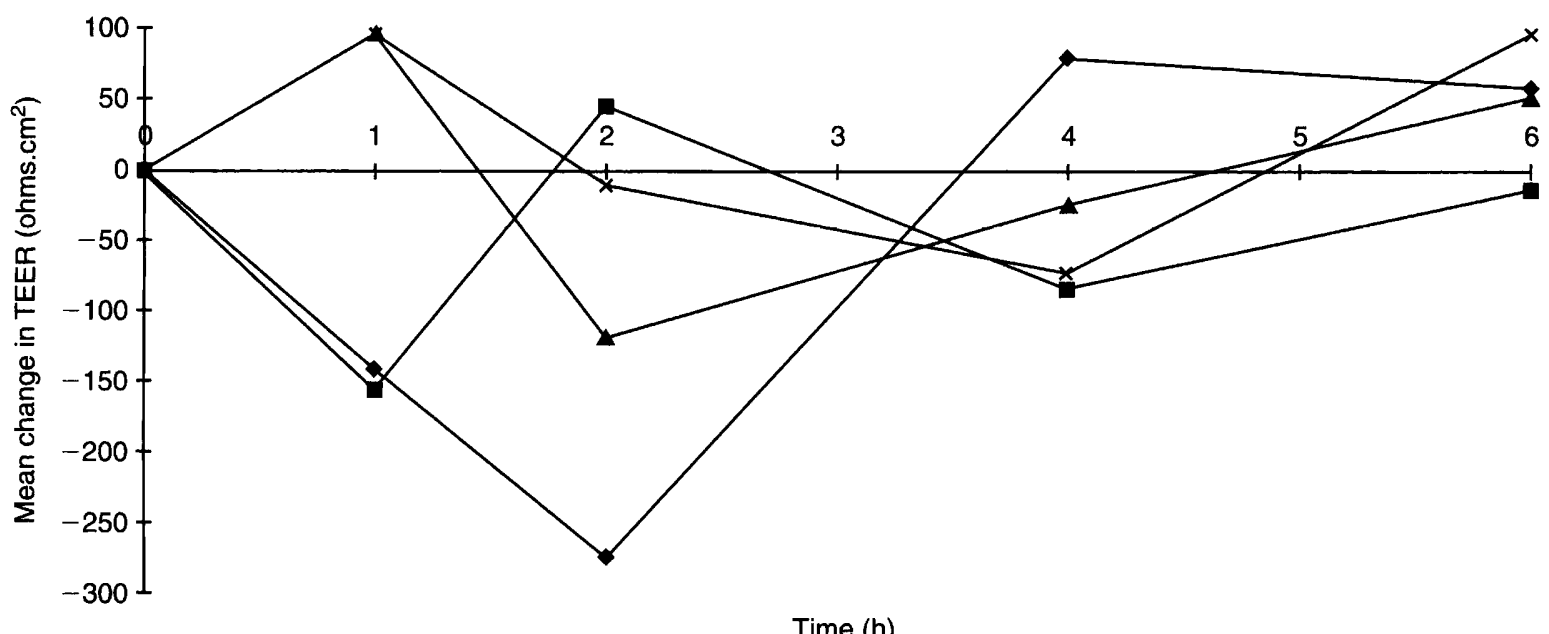

Fig. 3. Changes in TEER across polarised Caco-2 monolayers cultured on semi-permeable supports following infection with four different isolates of $C$. jejuni of contrasting invasiveness. Isolates: -- , no. 235 ; $-\rightarrow-$, no. $9519 ; \rightarrow-$, no. $9752 ;$, no. 10392. Data represent the mean of 18 replicate determinations from three separate experiments, corrected for the TEER of control uninfected monolayers.

smaller and less consistent fluctuations around the base-line TEER recorded across non-infected monolayers.

\section{Recovery of C. jejuni from within Caco-2 cells grown on permeable filters}

Higher proportions of $C$. jejuni isolates nos. 9519 and 10392 were recovered from within the monolayers than of isolates nos. 235 and 9752 (Fig. 4). The difference between isolates was statistically significant by the
Kruskal-Wallis test $(\mathrm{p}=0.002)$. Interestingly, with the more invasive isolates (nos. 9519 and 10392) the proportion of the inoculum recovered from within the monolayers on the Transwells was 10-fold higher than that previously recovered from the monolayers grown in traditional tissue-culture wells.

\section{Light microscopy of infected monolayers}

The Caco-2 cells adhered to the permeable filters, forming confluent monolayers. The cells exhibited a 


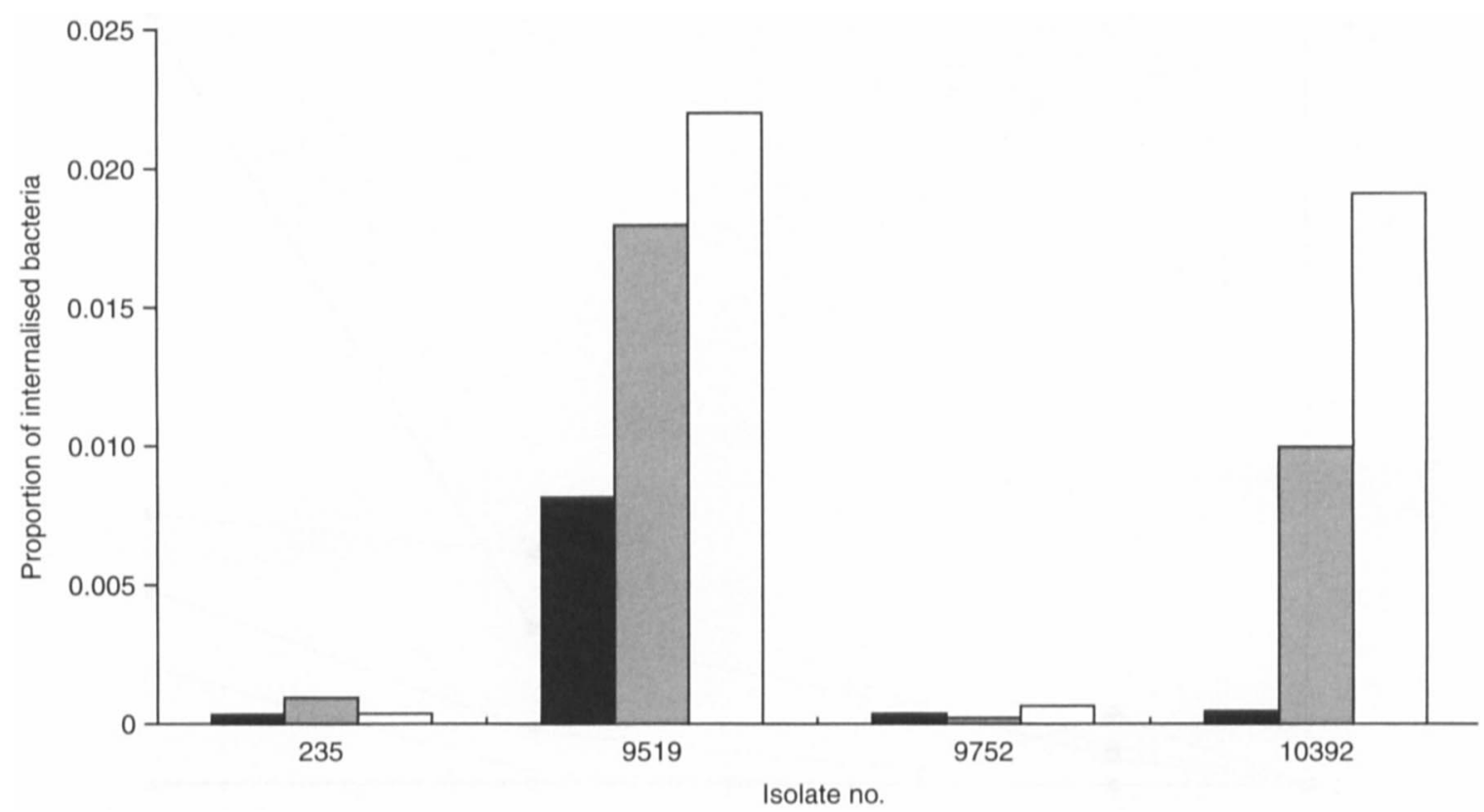

Fig. 4. Comparison of the intracellular invasion of Caco-2 cells cultured on semi-permeable supports by the four isolates of $C$. jejuni at $2 \mathrm{~h}(\boldsymbol{\square}), 4 \mathrm{~h}(\mathbf{0})$ and $6 \mathrm{~h}(\square)$. Data represent the proportion of the inoculum recovered from within the Caco-2 monolayer. Bars represent means (quadruplicate determinations). Differences between isolates were highly significant $(\mathrm{p}=0.002$, Kruskal-Wallis non-parametric analysis).

closely juxtaposed columnar morphology consistent with that described for polarised epithelium (Fig. 5), with a TEER of $c .400 \Omega \mathrm{cm}^{2}$.

Infection with isolate no. 235 produced no obvious alterations in the morphology of the Caco-2 cells at 2 or $6 \mathrm{~h}$. However, infection with isolate no. 9519 caused the Caco-2 cells to become flattened against the membrane of the Transwell unit at $2 \mathrm{~h}$. Cytoplasmic contraction and nuclear condensation were apparent at this stage, with an overall increase in the ratio of nuclear to cytoplasm volume. By $6 \mathrm{~h}$, the morphology of the monolayer had recovered to its original state. Monolayer confluency and integrity, as determined by light microscopy from the apical side, remained complete with both of the isolates throughout the assay period of $6 \mathrm{~h}$ (data not shown). This was supported by determinations of Caco-2 cell viability by Trypan blue staining and cell counting, which demonstrated that the proportions of viable Caco-2 cells recovered from the monolayers at each time point during infection with either isolate did not change significantly throughout.

\section{Discussion}

Campylobacters colonise the intestine following infection, before damage to epithelial cell function results in diarrhoea and, in many instances, acute inflammatory enteritis. Epithelial cell damage arises as a consequence of interactions between the pathogen and the epithelium, probably involving cell invasion or the production of toxins, or both $[8,19]$. A number of virulence determinants have been proposed for $C$. jejuni; those involved in the process of cell invasion are not fully understood, but probably involve flagella [21]. De novo bacterial protein synthesis is also thought to be involved [11], but the specific protein components have not been identified. The mechanisms by which campylobacters penetrate the epithelium are also unclear [22], but possibly involve interactions between the micro-organisms and the epithelial cells, promoting uptake by either microfilament-dependent [11] or microtubule-dependent processes [12], and the possible involvement of clathrin-coated pits [12] or caveolae, Gproteins and membrane ruffling [22].

The extent to which particular Campylobacter isolates invade Caco-2 cells has been reported to correlate with the severity of disease in man [18]. Strains isolated from patients with colitis were found to be more invasive than strains isolated from patients with watery diarrhoea. In the present study, the Campylobacter isolates also differed significantly in their invasiveness and in their kinetics of cell invasion, roughly dividing into two groups, one more invasive than the other.

C. jejuni isolates have also been shown previously to possess the capacity to translocate across polarised Caco-2 monolayers $[8,11,18]$. In separate electron microscopy studies, they have also been shown to gain access to junctional spaces between INT407 cells [12]. Correlations between the invasive ability of Campylobacter isolates and their potential to translocate across 


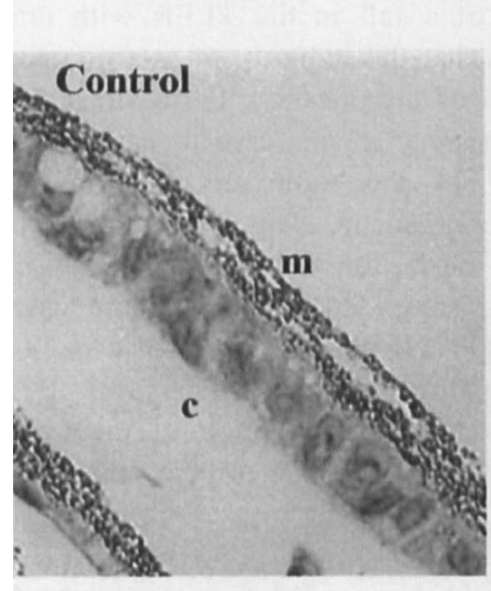

Isolate 235
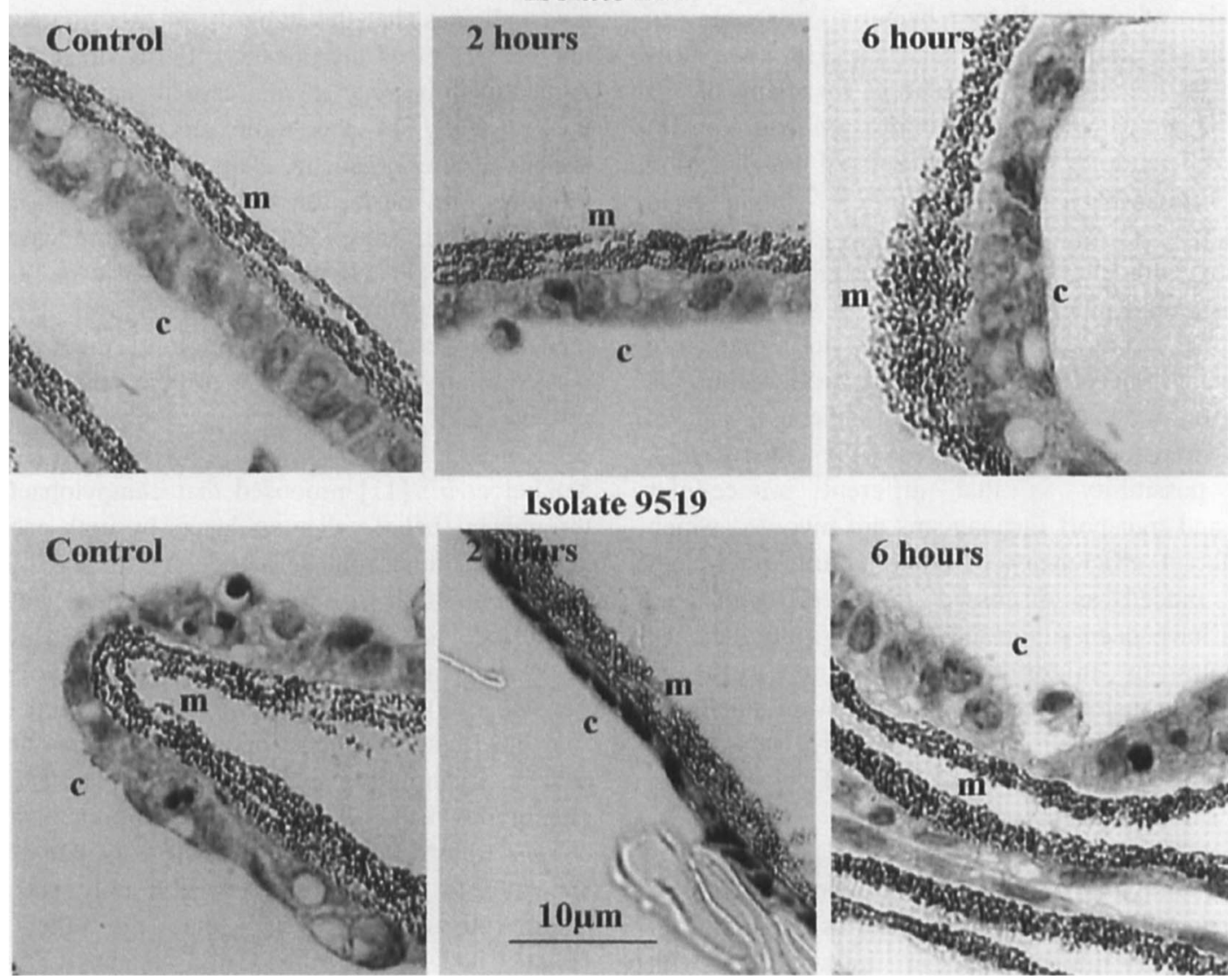

Isolate 9519
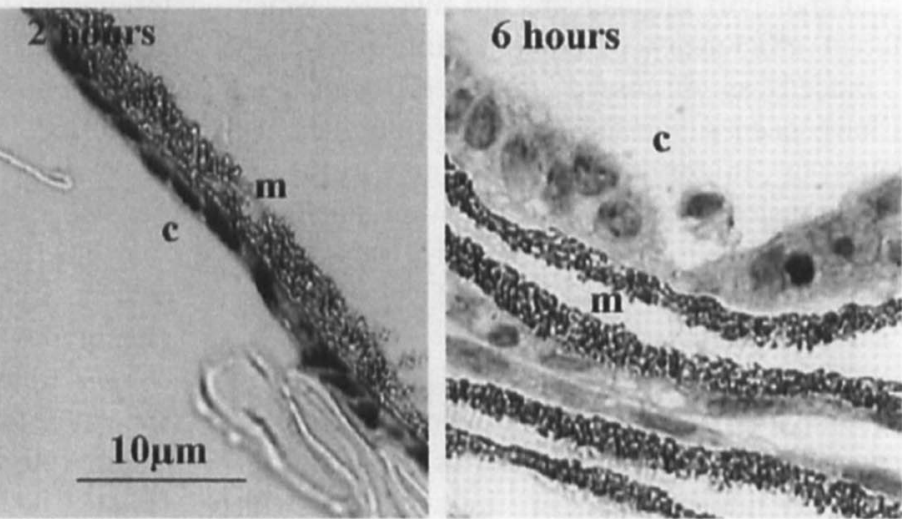

Fig. 5. Comparison of changes in Caco-2 cell morphology during translocation by C. jejuni isolates 235 and 9519 . Representative micrographs of sections across Transwell insert membranes (m) showing the typical columnar morphology of control and infected cells (c) in all cases except for isolate no. 9519 at $2 \mathrm{~h}$; in this case significant flattening of cells and condensation of nuclei in cells was observed.

epithelial cell monolayers have resulted from these studies, but comparative quantitative and kinetic data have been lacking. The relative contribution of cell invasion to monolayer translocation is, therefore, unclear.

The present study compared and contrasted the ability of $C$. jejuni isolates to translocate across polarised epithelial membranes with their ability to invade the same cell line. Four isolates with contrasting invasiveness were chosen. They varied significantly in their ability to translocate, but this did not correlate with their invasive ability.

All the C. jejuni isolates were detected in the lower chamber after incubation for $1 \mathrm{~h}$, as reported by Konkel et al. [11], and continued to translocate for up to $6 \mathrm{~h}$ as noted previously by Ketley [8]. Isolate no. 235 was found to be the most effective translocator, although it was identified in the earlier experiment as having poor invasive ability. Another poor invader, isolate no. 9752, showed only a modest ability to translocate. Interestingly, the highly invasive isolate no. 10392 was found to be a particularly poorly translocating strain, whilst isolate no. 9519, another highly invasive strain, was found to be able to translocate reasonably well. Konkel et al. [11] also demonstrated a similar spectrum of ability to translocate between their $C$. jejuni isolates, but their invasive ability was not compared.

Interestingly, the proportion of the $C$. jejuni inoculum recovered from within the monolayers during the transcytosis assay was considerably higher than that recovered from the standard invasion assay. This suggests that the physiological state of the Caco-2 cells was different when grown on the semi-permeable Transwell membranes, increasing their susceptibility to invasion. Changes in Caco-2 cell physiology have been reported previously to depend on cell age and culture conditions $[16,23]$. However, the distinction between the more invasive isolates (nos. 9519 and 10392) and the less invasive isolates (nos. 235 and 9752) remained the same. This demonstrated that the relative invasiveness of the isolates did not differ in the two assay formats and that factors other than invasion had contributed to the extent of translocation. This suggests that different isolates of Campylobacter penetrate the epithelial monolayers by different mechanisms, some of which involve intracellular 
invasion. An alternative explanation is that the different levels of intracellular bacteria are due to differences in intracellular survival. However, this seems to be an unlikely explanation for many of the observed discrepancies between invasion and translocation. For example, the high levels of translocation by the less invasive isolate no. 235, might have indicated poorer intracellular survival, yet in order to translocate intracellularly across the monolayers the organisms would have had to remain in a viable state. The reverse argument would apply to isolate no. 10392, which survived comparatively well within the monolayers, as determined by its invasiveness, and yet did not translocate well across the monolayers. Another possibility is that different intracellular invasion and transport mechanisms are involved which have different efficiencies of bacterial transfer across the cell. Indeed, as discussed above, different host pathways have been suggested involving, alternatively, microtubules or microfilaments. If more efficient intracellular translocation mechanisms were operating then this would result in fewer bacteria within the cells and an apparently poorer level of invasiveness, such as was seen with isolate no. 235 .

The proposition that different mechanisms of translocation are utilised by different isolates, either paracellular versus intracellular, or intracellular with different efficiencies of transfer, is further corroborated by the other responses of the Caco-2 monolayers with isolates nos. 235 and 9519 . The invasive isolate no. 9519 caused a large drop in the TEER at $2 \mathrm{~h}$ and light microscopy indicated a corresponding flattening of the Caco-2 cells. A similar response did not occur with the less invasive isolates no. 235. As there were no corresponding changes in Caco-2 cell viability, nor microscopic evidence of loss of monolayer confluency, it seems unlikely that isolate nos. 235 or 9519 were able to translocate because of degeneration of the monolayers. This supports the suggestion that isolate no. 9519 followed an intracellular pathway involving invasion and cytoskeletal re-arrangement, resulting in alterations in cell shape. This may have contributed to some disruption of tight junction integrity, as indicated by the drop in TEER. Alternatively, the integrity of the tight junctions may have remained unaffected. The drop in TEER may be a consequence of a disruption to a transcellular pathway affecting ion transport mechanisms, as has been suggested to be the case with enteropathogenic E. coli [6].

In contrast, isolate no. 235 caused no consistent changes in TEER or cell morphology. A similar lack of damage following infection with another $C$. jejuni isolate, M129, was reported in a previous study [11]. It seems likely that isolate no. 235 translocated across the epithelial cell monolayers due to movement through the tight junctions, i.e., a paracellular route, or alternatively a more efficient intracellular pathway involving less disruption to the cytoskeleton. The relative lack of a fall in the TEER with this isolate also indicates that the integrity of the monolayers was not compromised significantly. If translocation was by paracellular passage in this case it may have involved the resealing of the tight junctions following the transit of the organism. This phenomenon has been reported previously for Salmonella typhimurium [24] and has been suggested also to be the case for $C$. jejuni M129 [11]. If the alternative of a more efficient intracellular pathway was involved with this isolate then a lower level of disruption of the cytoskeleton may similarly be responsible for the relative lack of a fall in TEER.

Konkel et al. [11] proposed that campylobacters pass through epithelial cell monolayers by both paracellular and intracellular routes. Based on this and the results of other studies, separate phenotypes have been suggested to exist amongst clinical isolates of Campylobacter [8], including non-invasive (without translocation), invasive (with translocation) and invasive without translocation. The data presented here provide quantitative evidence to substantiate distinct phenotypes. Isolate no. 9752 showed poor invasive and modest translocation ability, whereas isolate no. 10392 appeared to penetrate by invasion only and translocated 100 times less well than the other isolates. Isolate no. 9519 translocated modestly and also invaded well. However, isolate no. 235 translocated very efficiently with evidence of much poorer intracellular invasion. These levels of invasion and translocation were comparable to those described in earlier studies $[11,25]$.

In summary, this study confirmed that isolates of $C$. jejuni differ significantly in their interactions with epithelial cells both in terms of their ability to invade Caco-2 cells on solid supports and to translocate across polarised Caco-2 monolayers supported on semi-permeable membranes. By comparing these two attributes carefully with four contrasting isolates it has been shown for the first time that invasiveness does not correlate quantitatively with epithelial translocation for this pathogen. This provides further evidence for the proposition that the invasiveness of phenotypes of $C$. jejuni may differ significantly and possibly involve different extents of paracellular or intracellular penetration, or translocation mechanisms that differ in their efficiency. This is further suggested by the fact that two of the isolates differed in their effects on the morphology and TEER of the monolayers. This is the first report of any change in these parameters being caused by $C$. jejuni. It will be interesting to study these different invasion phenotypes of $C$. jejuni further, as they are likely to contribute to different disease manifestations in the host.

This work was funded by the Department of Health, London. We would like to acknowledge the technical assistance of Paula Freemantle, John Latigo and Celine Touchard for histology and 
tissue culture. Caco-2 cells were kindly provided by ECACC Encouragement and help with the manuscript was kindly provided by Professor Philip Marsh.

\section{References}

1. Polotsky Y, Dragunsky E, Khavkin T. Morphologic evaluation of the pathogenesis of bacterial enteric infections. Crit Rev Microbiol 1994; 20: 161-208.

2. Falkow S, Isberg RR, Portnoy DA. The interactions of bacteria with mammalian cells. Annu Rev Cell Biol 1992; 8: $333-363$.

3. Siebers A, Finlay BB. Models to study enteropathogenic bacteria: lessons learned from Shigella. Trends Microbiol 1995; 3: 207-209.

4. Kernéis S, Bogdanova A, Kraehenbuhl J-P, Pringault E. Conversion by Peyer's patch lymphocytes of human enterocytes into M cells that transport bacteria. Science 1997; 277: 949-952.

5. Finlay BB, Falkow S. Salmonella interactions with polarized human intestinal Caco-2 epithelial cells. J Infect Dis 1990; 162: $1096-1106$

6. Canil C, Rosenshine I, Ruschkowski S, Donnenberg MS, Kaper JB, Finlay BB. Enteropathogenic Escherichia coli decreases the transepithelial electrical resistance of polarized epithelial monolayers. Infect Immun 1993; 61: 2755-2762.

7. Czuprynski CJ, Balish E. Pathogenesis of Listeria monocytogenes for gnotobiotic rats. Infect Immun 1981; 32: 323-331.

8. Ketley JM. Virulence of Campylobacter species: a molecular genetic approach. J Med Microbiol 1995; 42: 312-327.

9. Allos BM, Blaser MJ. Campylobacter jejuni and the expanding spectrum of related infections. Clin Infect Dis 1995; 20: 1092 1101.

10. Russell RG, Blaser MJ, Sarmiento JL, Fox J. Experimental Campylobacter jejuni infection in Macaca nemestrina. Infect Immun 1989; 57: 1438-1444.

11. Konkel ME, Mead DJ, Hayes SF, Cieplak W. Translocation of Campylobacter jejuni across human polarized epithelial cell monolayer cultures. J Infect Dis 1992; 166: 308-315.

12. Oelschlaeger TA, Guerry P, Kopecko DJ. Unusual microtubuledependent endocytosis mechanisms triggered by Campylobacter jejuni and Citrobacter freundii. Proc Natl Acad Sci USA
1993; 90: 6884-6888.

13. Konkel ME, Joens LA. Adhesion to and invasion of HEp-2 cells by Campylobacter spp. Infect Immun 1989; 57: 29842990.

14. de Melo MA, Gabbiani G, Pechère J-C. Cellular events and intracellular survival of Campylobacter jejuni during infection of HEp-2 cells. Infect Immun 1989; 57: 2214-2222.

15. Grant CRC, Konkel ME, Cieplak W, Tompkins LS. Role of flagella in adherence, internalization, and translocation of Campylobacter jejuni in nonpolarized and polarized epithelial cell cultures. Infect Immun 1993; 61: 1764-1771.

16. Hidalgo IJ, Raub TJ, Borchardt RT. Characterization of the human colon carcinoma cell line (Caco-2) as a model system for intestinal epithelial permeability. Gastroenterology 1989; 96: $736-749$.

17. Conte MP, Longhi $\mathrm{C}$, Polidoro $\mathrm{M}$ et al. Iron availability affects entry of Listeria monocytogenes into the enterocyte cell line Caco-2. Infect Immun 1996; 64: 3925-3929

18. Everest $\mathrm{PH}$, Goossens $\mathrm{H}$, Butzler J-P et al. Differentiated Caco-2 cells as a model for enteric invasion by Campylobacter jejuni and C. coli. J Med Microbiol 1992; 37: 319-325.

19. Ketley JM. Pathogenesis of enteric infection by Campylobacter. Microbiology 1997; 143: 5-21.

20. Leach S, Harvey $P$, Wait $R$. Changes with growth rate in the membrane lipid composition of and amino acid utilization by continuous cultures of Campylobacter jejuni. J Appl Microbiol 1997; 82: $631-640$.

21. Wassenaar TM, Bleumink-Pluym NMC, van der Zeijst BAM. Inactivation of Campylobacter jejuni flagellin genes by homologous recombination demonstrates that flaA but not fla $\beta$ is required for invasion. EMBO $J$ 1991; 10: 2055-2061.

22. Wooldridge KG, Williams PH, Ketley JM. Host signal transduction and endocytosis of Campylobacter jejuni. Microb Pathog 1996; 21: 299-305.

23. Engle MJ, Goetz GS, Alpers DH. Caco-2 cells express a combination of colonocyte and enterocyte phenotypes. $\mathrm{J}$ Cell Physiol 1998; 174: 362-369.

24. Takeuchi A. Electron microscope studies of experimental Salmonella infection. I. Penetration into the intestinal epithelium by Salmonella typhimurium. Am J Pathol 1967; 50: 109136.

25. Szymanski CM, King M, Haardt M, Armstrong GD. Campylobacter jejuni motility and invasion of Caco-2 cells. Infect Immun 1995; 63: 4295-4300. 